\title{
Increased Expression of Microglial Markers in the Arcuate Nucleus of Mice Exposed to Dim Light at Night
}

\author{
C. Wyse ${ }^{1}$, L. Desbonnet ${ }^{2}$, N. Kangasmaa ${ }^{1}$, A. Russell ${ }^{1}$ and Biello $\mathrm{SM}^{3}$ \\ ${ }^{1}$ Institute of Biodiversity, Animal Health and Comparative Medicine, University of Glasgow, G61 $1 Q H$ and ${ }^{3}$ Institute of \\ Neuroscience and Psychology, University of Glasgow, G12 8QB
}

There is an increased prevalence of obesity and cardiometabolic disease in human shift-workers that might be partly mediated by disruption of circadian rhythms. ${ }^{(1)}$ Studies in both humans and animals have reported that disruption of circadian rhythms affects glucose homeostasis, ${ }^{(2-3)}$ and increases body weight. ${ }^{(3)}$ Circadian disruption (CD) is classically associated with shiftwork, but a more chronic form of disruption induced by the irregular lifestyle and artificial light of the urban environment could similarly disrupt rhythmicity and affect metabolic health. Inflammation and activation of microglia in the hypothalamus is thought to contribute to the pathogenesis of obesity, ${ }^{(4)}$ and upregulation of inflammatory signalling was also associated with CR induced by dim light at night ${ }^{(5)}$. The aim of this study was to investigate the effects of chronic dim light at night on body weight, food intake and markers of glial plasticity in the hypothalamus, and to assess if social interaction by group housing modulates any effects.

Adult mice $(\mathrm{n}=32)$ were housed for 10 weeks in either dim light at night (12D:12L at 5 lux) or control conditions of a 12:12 light dark cycle. The animals were further allocated to be housed singly, or in groups of 4 . After 10 weeks, the animals were sacrificed and their brains processed for detection of markers of microglia (Iba1) in the arcuate nucleus of the hypothalamus using immunohistochemistry. Confocal microscopy was used to acquire images of the hypothalamus and immunopositivity was quantified using ImageJ and normalised to the area of interest in the arcuate nucleus region. Circadian rhythmicity was monitored using passive infra red sensors attached to the cage tops to monitor locomotor activity at 1 minute intervals for periods of 10 days, and body weight and food intake were monitored every week.

\begin{tabular}{llll}
\hline \multicolumn{4}{c}{ \% Increase in Body Weight } \\
\hline \multirow{4}{*}{ Control } & & Single & Group \\
& & Housed & Housed \\
& Mean & 8.58 & 9.25 \\
& SE & 0.58 & 0.71 \\
& Mean & 8.91 & 8.92 \\
& SE & 0.64 & 0.38
\end{tabular}
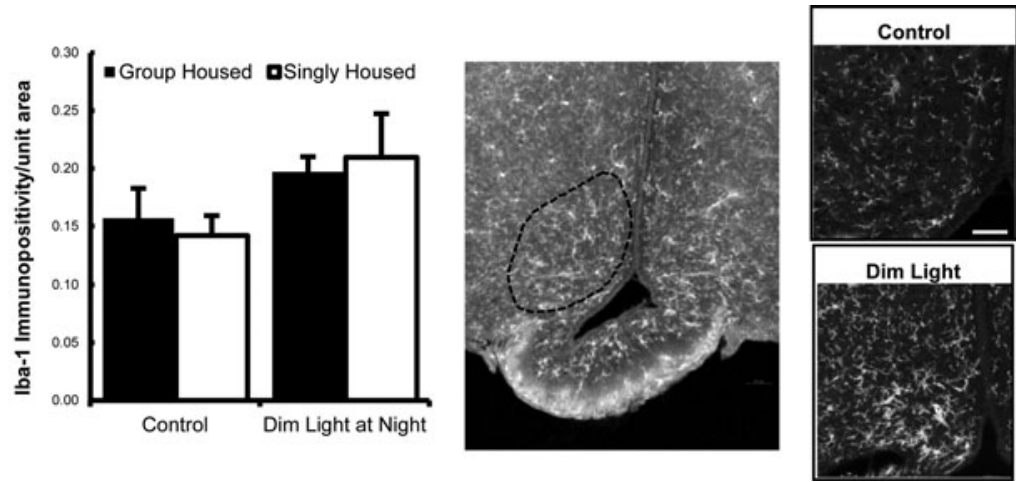

The mice exposed to dim light at night showed disruption of circadian rhythms with more day time activity and less consolidated activity at night. There was no effect of either housing or photoperiod on body weight; expression of microglial markers in the arcuate nucleus was significantly increased $(\mathrm{p}<0.05)$ in the dim light group, but was not affected by housing $(\mathrm{p}<0.05)$. Dim light was associated with increased microglia in the hypothalamus in this study, although body weight was not affected. These findings are further evidence for an effect of CD on central inflammatory signalling, and suggest that this mechanism might precede the changes in body weight associated with disruption of rhythms.

1. Karlsson B, Knutsson A, Lindahl B et al. (2001) Occup Environ Med 58, 747-52.

2. Scheer FA, Hilton MF, Mantzoros CS, et al. (2009) Proc Natl Acad Sci 106, 4453-8.

3. Karatsoreos IN, Bhagat S, Bloss EB, et al. (2011) Proc Natl Acad Sci 108, 1657-62.

4. Thaler JP, Yi X, Schur EA, Guyenet SJ, et al. (2012) J Clin Invest 122, 153-162.

5. Fonken LK, Lieberman RA, Weil ZM, et al. (2013) Endocrinology 154, 3817-25. 